\title{
An in-silico Analysis of the Ability of Dynamic Tests to Trace the Kinetic Behaviour of Insulin Sensitizer Drugs
}

\author{
Paul D Docherty *, J Geoffrey Chase *, Thomas F Lotz *, \\ Juliet E Berkeley ** and Geoffrey M Shaw **
}

\author{
*Centre for Bioengineering, University of Canterbury, New Zealand \\ (Tel:0064-3-364-2987-ex7486,e-mail: paul.docherty@pg.canterbury.ac.nz). \\ **Christchurch School of Medicine, University of Otago, New Zealand \\ (e-mail:Juliet.berkeley@cdhb.govt.nz)
}

\begin{abstract}
A Monte Carlo analysis was undertaken to measure the ability of a series of dynamic insulin sensitivity and secretion tests (DISST) to observe and quantify the time-varying effect of an insulin sensitizer drug. Physiological parameter values from an insulin resistant individual were used to simulate a series of DISST tests with the effects a hypothetical sensitizer drug (based on Metformin) that was assumed to elevate insulin sensitivity $(D)$ by $50 \%$, and have absorption $\left(D k_{l}\right)$ and decay $\left(D k_{2}\right)$ half-lives of $\sim 30$ and $\sim 140$ minutes respectively. Noise was added to data sampled from the simulation and allowed repeated identification of pharmaco-kinetic/dynamic parameters in clinically realistic data. The coefficients of variation $(\mathrm{CV})$ of the drug variables in this Monte Carlo analysis were CV- $D=0.9 \%, \mathrm{CV}$ $D k_{1}=116.3 \%$, and CV- $D k_{2}=41.4 \%$ respectively. Although the CV values for the drug kinetic rates did not indicate considerable stability, the identified time-varying insulin sensitivity profile was relatively accurate to the simulation profile (median error of $0.047 \mathrm{~L} / \mathrm{mU} / \mathrm{min}(\sim 2 \%)$ and IQR of -0.093 to 0.184 $\mathrm{L} / \mathrm{mU} / \mathrm{min}(-4 \%$ to $8 \%))$. This result indicates that the proposed method for identifying drug parameters using a series of dynamic tests is able to capture the overall effect of the drug, but has a potentially limited ability to identify the drug parameters individually. Thus, the existing method of arduous, frequently-sampled steady-state tests for the measurement of drug pharmacokinetics and dynamics could be replaced with a series of sparsely-sampled dynamic tests.
\end{abstract}

Keywords: Physiological modeling, pharmacokinetics/dynamics, parameter-identification, insulin sensitivity.

\section{INTRODUCTION}

Insulin sensitizer drugs are used in treating type 2 diabetes mellitus to minimise the incidence of hyperglycaemia in individuals who require aid to effectively regulate their blood glucose concentration. Although there is a reasonable quantity of studies investigating the long term effects of such drugs, there are a limited number of studies investigating the drugs' single dose kinetics. Typically, the effect of these drugs are measured by the companies that produce them using arduous steady-state clamp tests that last the duration of the drug's efficacy. These steady-state tests require five or ten minutely samples to enable feedback control for euglycemia. This approach is thus very costly, time consuming and intensive.

This study investigates a series of sparsely sampled dynamic tests as a possible alternative for this clinically intense approach. Our group has previously presented the dynamic insulin sensitivity and secretion test (DISST) as a low-cost, comparatively low intensity, but relatively high resolution insulin sensitivity test that also measures the participants endogenous insulin production response (Lotz et al. 2010). It is hypothesised that a series of these dynamic tests may enable an observation of the change in effect of these drugs over time. To test this hypothesis, an in-silico Monte Carlo analysis is completed that simulates the expected level of clinical assay error and measures the ability of the novel identification methods to reproduce values of a theoretical sensitizer drug's pharmaco-kinetics (PK) and pharmacodynamics (PD).

\section{METHODS}

\subsection{Virtual Participant}

The parameters used to construct the virtual participant of this study were obtained from a participant of the DISST pilot study (Lotz 2007, Lotz et al. 2010). This particular participant was very insulin resistant with suspected undiagnosed type 2 diabetes mellitus. The participant had a 


\begin{tabular}{|c|c|c|c|c|c|c|c|c|c|}
\hline \multirow[b]{2}{*}{ Sex } & \multirow[b]{2}{*}{ Age } & \multirow{2}{*}{$\begin{array}{c}\text { BMI } \\
{\left[\mathrm{kg} \cdot \mathrm{m}^{-2}\right]}\end{array}$} & \multicolumn{3}{|c|}{$\boldsymbol{U}_{n}\left[\mathrm{mU} \cdot \mathrm{min}^{-1}\right]$} & \multicolumn{2}{|c|}{ Insulin clearance } & \multirow{2}{*}{$\begin{array}{l}\mathbf{V}_{\mathbf{G}} \\
{[\mathrm{L}]}\end{array}$} & \multirow{2}{*}{$\begin{array}{c}\text { SI } \\
{[\mathrm{L} / \mathrm{mU} / \mathrm{min}]}\end{array}$} \\
\hline & & & $U_{b}$ & $U_{1}$ & $U_{2}$ & $\boldsymbol{n}_{\boldsymbol{L}}\left[\mathrm{min}^{-1}\right]$ & $x_{L}[1]$ & & \\
\hline $\mathrm{F}$ & 57 & 33.9 & 115.5 & 233.6 & 150.7 & 0.064 & 0.822 & 13.35 & 2.236 \\
\hline
\end{tabular}

Table 1. Key parameters of the participant used to generate the virtual participant used in this study, where all terms are defined in Table 2 except for $U_{b}, U_{l}$ and $U_{2}$ which represent the basal, first and second phases of insulin production.

significant insulin production rate, but a relative inability to effectively clear glucose. Thus, she could be the type of person who might gain an advantage from insulin sensitizer treatment, and is likely to represent the physiology of patients already on insulin sensitizer treatment. Some key anatomical and PK/PD parameters for this participant are summarised in Table 1.

\subsection{Model Equations}

This study will use a previously validated physiological model (Lotz et al. 2010), defined:

$$
\begin{aligned}
& \dot{C}(t)=k_{2} Y(t)-\left(k_{1}+n_{k}\right) C(t)+\frac{U_{n}(t)}{V p} \\
& \dot{Y}(t)=k_{1} C(t)-k_{2} Y(t) \\
& \dot{I}(t)=-n_{k} I(t)-n_{L} \frac{I(t)}{1+\alpha_{I} I(t)} \ldots \\
& \ldots-\frac{n_{I}}{V p}(I(t)-Q(t))+x_{L} U_{n}(t)+\frac{U_{X}(t)}{V p} \\
& \dot{Q}(t)=\frac{n_{I}}{V q}(I(t)-Q(t))-n_{C} Q(t) \\
& \dot{G}(t)=-p_{G}\left(G(t)-G_{b}\right) \ldots \\
& \ldots-S I\left(G(t) Q(t)-G_{b} Q_{b}\right)+\frac{P_{X}(t)}{V_{G}}
\end{aligned}
$$

where: equation nomenclature is defined in Table 2.

For this analysis, saturation of hepatic insulin clearance is assumed to be negligible and thus, $a_{I}$ is set to zero and $n_{L}$ and $n_{K}$ are combined to a single parameter of insulin clearance from plasma $\left(n_{T}\right)$.

If it is assumed that the drug is administered orally or subcutaneously, it would be reasonable to propose a very simple two-compartment model for the PKs of the theoretical insulin sensitizer drug. It is assumed for the purpose of this preliminary, proof-of-concept investigation, that the transport between compartments will be concentration-based and the drug will not re-enter the remote compartment from the active compartment. Figure 1 and Equations (6)-(8) define the model used in this study and the effect of the drug on

\begin{tabular}{|c|c|c|}
\hline Sym' & Definition & units \\
\hline$C$ & Plasma C-peptide concentration & $\mathrm{mU} / \mathrm{L}$ \\
\hline$Y$ & Interstitial C-peptide conc. & $\mathrm{mU} / \mathrm{L}$ \\
\hline$k_{1}$ & Transport rate to interstitial & $1 / \min$ \\
\hline$k_{2}$ & C-peptide transport rate to plasma & $1 / \mathrm{min}$ \\
\hline$n_{K}$ & $\begin{array}{l}\text { Kidney clearance of plasma insulin } \\
\text { and C-peptide }\end{array}$ & $1 / \mathrm{min}$ \\
\hline$U_{n}$ & $\begin{array}{l}\text { Endogenous insulin production rate } \\
\text { profile }\end{array}$ & $\mathrm{mU} / \mathrm{L} / \mathrm{min}$ \\
\hline I & Plasma insulin concentration & $\mathrm{mU} / \mathrm{L}$ \\
\hline$Q$ & Interstitial insulin concentration & $\mathrm{mU} / \mathrm{L}$ \\
\hline$n_{L}$ & Hepatic insulin clearance & $1 / \min$ \\
\hline$a_{I}$ & Saturation of hepatic clearance & $\mathrm{L} / \mathrm{mU}$ \\
\hline$n_{I}$ & $\begin{array}{l}\text { Transition of insulin between plasma } \\
\text { and interstitial }\end{array}$ & $\mathrm{L} / \mathrm{min}$ \\
\hline$x_{L}$ & First pass clearance of insulin & 1 \\
\hline$V p$ & Plasma insulin distribution volume & $\mathrm{L}$ \\
\hline$V q$ & $\begin{array}{l}\text { Interstitial insulin distribution } \\
\text { volume }\end{array}$ & $\mathrm{L}$ \\
\hline$G$ & Glucose concentration & $\mathrm{mmol} / \mathrm{L}$ \\
\hline$G_{b}$ & Basal glucose concentration & $\mathrm{mmol} / \mathrm{L}$ \\
\hline$Q_{b}$ & Basal interstitial insulin conc. & $\mathrm{mU} / \mathrm{L}$ \\
\hline$p_{G}$ & 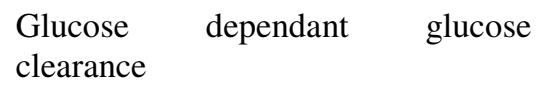 & $1 / \min$ \\
\hline$S I$ & Insulin sensitivity & $\mathrm{L} / \mathrm{mU} / \mathrm{min}$ \\
\hline$P_{X}$ & Exogenous glucose bolus & mmol \\
\hline$V_{G}$ & Glucose distribution volume & $\mathrm{L}$ \\
\hline$S$ & $\begin{array}{l}\text { Latent drug effect in remote } \\
\text { compartment }\end{array}$ & 1 \\
\hline$P$ & Drug effect in active compartment & 1 \\
\hline$D$ & $\begin{array}{l}\text { Total potential proportional effect of } \\
\text { drug bolus on } S I\end{array}$ & 1 \\
\hline$D k_{1}$ & $\begin{array}{l}\text { Passive transport rate from remote to } \\
\text { active compartments. }\end{array}$ & $1 / \mathrm{min}$ \\
\hline$D k_{2}$ & $\begin{array}{l}\text { Passive clearance from active } \\
\text { compartment. }\end{array}$ & $1 / \mathrm{min}$ \\
\hline$S I_{0}$ & Basal insulin sensitivity & 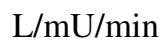 \\
\hline$S I(t)$ & Time variant insulin sensitivity & $\mathrm{L} / \mathrm{mU} / \mathrm{min}$ \\
\hline
\end{tabular}
insulin sensitivity:

$$
\begin{aligned}
& \dot{S}(t)=D(t)-D k_{1} S(t) \\
& \dot{P}(t)=D k_{1} S(t)-D k_{2} P(t) \\
& S I(t)=S I_{0}(1+P(t))
\end{aligned}
$$

where: Equation nomenclature is defined in Table 2
Table 2. Nomenclature from Equations (1)-(8).

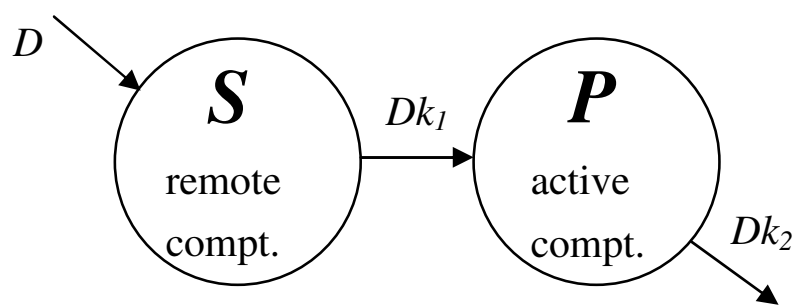

Figure 1. Two compartment representation of the PKs of a theoretical sensitizer drug. 


\subsection{Proposed Protocol}

The effect of a theoretical insulin sensitizer drug during a series of DISST tests will be simulated in a virtual participant over the 12 hours. The PKs of the theoretical drug used in this study are based on those of Metformin (Pentikäinen et al. 1979). Seven DISST tests will begin at two hour intervals with blood samples taken at $\mathrm{t}=0,1025$ and 40 minutes in each test. Thus, the total length of this virtual simulation is 760 minutes (12 hours, 40 minutes), and the total number of samples is 28. Each of the seven DISST tests requires $10 \mathrm{~g}$ glucose and $1 \mathrm{U}$ insulin (intravenous) bolus immediately after the $\mathrm{t}=0$ and 10 minute samples respectively. The sensitizer drug is administered at $\mathrm{t}=150$ minutes toward the end of the second test. Hence the first two tests serve as a baseline so each subject is their own control. All samples are assayed for insulin, C-peptide and glucose.

\subsection{Parameter Identification}

This analysis will define $U_{n}, n_{T}, x_{L}, S I_{0}, V_{G}, D, D k_{l}$ and $D k_{2}$ as variables to be identified. All other model parameters are assumed to be known through a-priori means (Lotz 2007, Van Cauter et al. 1992).

$U_{n}, n_{T}, x_{L}, S I_{O}$ and $V_{G}$ are identified using previously presented methods: $U_{n}$ is identified using a typical deconvolution approach (Van Cauter et al. 1992), $n_{T}, x_{L}, S I_{O}$ and $V_{G}$ are identified using the iterative integral method (Docherty et al. 2009, Hann et al. 2005), $S I_{0}$ and $V_{G}$ are identified using only data from the first 150 minutes of the proposed protocol and are then held constant.

To identify the PK/PD of the sensitizer drugs, a comprehensive model of the glucose PDs must be generated. Combining Equations (5) and (8) provides:

$$
\begin{aligned}
& \dot{G}(t)=-p_{G}\left(G(t)-G_{0}\right) \ldots \\
& \quad \ldots-S I_{0}(1+P(t)) G(t) Q(t)+S I_{0} G_{0} Q_{0}+\frac{P_{X}(t)}{V_{G}}
\end{aligned}
$$

where:

$$
P(t)=D k_{1} e^{-D k_{2} t} \int_{0}^{t}\left(e^{\left(D k_{2}-D k_{1}\right)} t \int_{0}^{t} e^{D k_{1} t} D(t) d t\right) d t
$$

The variables in Equations (9) and (10) are not separable in terms of the glucose data and as such the iterative integral method is not possible. To enable variable identification, an approximation of the $P(t)$ profile must first be de-convolved from Equation (9). Equation (9) can be rearranged for $P(t)$ :

$$
\begin{aligned}
& P(t)_{\text {approx }}=\cdots \\
& \frac{-\left(\dot{G}(t)+p_{G}\left(G(t)-G_{0}\right)-S I_{0} G_{0} Q_{0}-\frac{P_{X}(t)}{V_{G}}\right)}{S I_{0} G(t) Q(t)}-1
\end{aligned}
$$

With the exception of $G(t)$ all parameters of Equation (9) are known or have been identified. $G(t)$ must be approximated with the highest possible accuracy following the drug administration. Steps 1-4 outline this process:
1. Simulate a preliminary $G(t)$ for the full duration of all tests. Initially, this is done using the values for $S I_{0}$ and $V_{G}$ identified with the iterative integral method and the baseline data from the first 150 minutes of the test, and then using $S I(t)$ and $V_{G}$ $\left(G(t)_{\text {prelim }}\right)$.

2. Define a linear interpolation between the measured data points $\left(G(t)_{\text {interp }}\right)$

3. Define a linear interpolation between the values of $G(t)_{\text {prelim }}$ at the sample times $\left(G(t)_{\text {preint }}\right)$

4. The difference between $G(t)_{\text {interp }}$ and $G(t)_{\text {preint }}$ is attributable to the effect of the sensitizer and thus Equation (12) can be used as an approximation of $G(t)$ :

$$
G(t)=G(t)_{\text {prelim }}+G(t)_{\text {interp }}-G(t)_{\text {preint }} .
$$

With an approximation of $G(t), P(t)_{\text {approx }}$ can be evaluated in Equation (11). This profile is then used with Equation (10) in a non-linear least square Levenberg-Marquardt parameter identification method to find the values of $D k_{1}, D k_{2}$ and $D$ that minimise the function in Equation (13).

$$
\text { error }=\left\|P(t)_{\text {approx }}-P(t)_{\text {eq10 }}\right\|_{2}
$$

Once the PK/PD values of Equation (13) have converged sufficiently, $P(t)$ is re-evaluated using Equation (10). Thus, $G(t)$ can be re-simulated, and steps 1-4 and the LevenbergMarquardt parameter identification process can be iterated. In total, 5 iterations are used, by which time parameter convergence is on the order of $1 \%$.

\subsection{Concept Evaluation}

A Monte Carlo analysis is used to assess the ability of the identification method and proposed protocol to detect and quantify the PKs of the theoretical sensitizer drug. C-peptide, insulin and glucose concentration profiles are simulated using Equations (1)-(4), and (9) with the protocol from Section 2.3.

The participant parameter values used in the simulation are shown in Table 1. The theoretical drug kinetics are based on those of Metformin (Pentikäinen et al. 1979): $D k_{l}$ is defined as $0.005 / \mathrm{min}$, representing an absorption half-life of $\sim 140$ minutes. Similarly $D k_{2}$ is defined as $0.0015 / \mathrm{min}$, representing a clearance half-life of $\sim 460$ minutes. Finally, $D$ is defined as 0.5 , meaning a $50 \%$ increase in insulin sensitivity could be expected if the full amount of the drug dose was in the active compartment. The three C-peptide, insulin and glucose profiles are 'sampled' at the prescribed times and this represents a noiseless set of data measurements.

The Monte-Carlo simulation identifies the eight variables mentioned at the start of Section 2.4 a total of 1000 times using the method described. Each iteration has normallydistributed random noise added to the noiseless glucose, insulin and C-peptide data sets. The magnitude of the added noise is in accordance with realistic clinical assay error (glucose: $\mathrm{CV}=2 \%$, insulin: $\mathrm{CV}=3 \%$, and C-peptide: $\mathrm{CV}=4 \%$ to a maximum of three standard deviations). 
The median and variation of the identified variables of Equations (9)-(10) are presented. Furthermore, the median and inter-quartile range (IQR) of the $S I(t)$ re-simulations will be compared to the noiseless simulation of Equation (8) to assess the ability of the method to track the PDs of the drug.

\section{RESULTS}

Table 3 summarises the variation in the identified variables that define the drug PK/PDs in Equations (9)-(10).

\begin{tabular}{rccc}
\hline & $\begin{array}{c}\text { True } \\
\text { Value }\end{array}$ & $\begin{array}{c}\text { Mean } \\
(\mathbf{C V})\end{array}$ & $\begin{array}{c}\text { Median } \\
(\text { IQR })\end{array}$ \\
\hline $\boldsymbol{S I}$ & 2.236 & $\begin{array}{c}2.252 \\
(7.9 \%)\end{array}$ & $\begin{array}{c}2.240 \\
(2.112-2.355)\end{array}$ \\
{$[\mathrm{L} / \mathrm{mU} / \mathrm{min}]$} & & 13.36 & 13.31 \\
$\boldsymbol{V} \boldsymbol{g}$ & \multirow{2}{*}{13.35} & $(4.5 \%)$ & $(12.95-13.74)$ \\
{$[\mathrm{L}]$} & & 0.498 & 0.500 \\
$\boldsymbol{D}$ & \multirow{2}{*}{0.5} & $(0.9 \%)$ & $(0.498-0.500)$ \\
{$[1]$} & & 0.0103 & 0.0060 \\
$\boldsymbol{D} \boldsymbol{k}_{\boldsymbol{l}}$ & 0.005 & $(116.3 \%)$ & $(0.0048-0.0086)$ \\
{$[1 / \mathrm{min}]$} & & 0.0013 & 0.0013 \\
$\boldsymbol{D} \boldsymbol{k}_{\mathbf{2}}$ & 0.0015 & $(41.4 \%)$ & $(0.0010-0.0017)$ \\
{$[1 / \mathrm{min}]$} & &
\end{tabular}

Table 3. The effect of noise on variable identification.

Figure 2 shows the range of time-varying sensitivity profiles identified by the method described in Section 2.4. It can be seen that the median profile is approximately equal to the true value for the duration of the test. The identification method generally seems to slightly overestimate the drug absorption rate $\left(D k_{l}\right)$. However the $100^{\text {th }}$ percentile simulation shows that at some outliers drastically overestimate the absorption rate. These values must contribute to the higher than expected mean and CV for $D k_{l}$. The median and IQR of $D k_{l}$ indicates that the values typically identified are within expected ranges.
Table 4 summaries the residuals of the time varying insulin sensitivity profiles shown in Figure 2.

\begin{tabular}{rc}
\hline & Median $(\mathrm{IQR})[\mathrm{L} / \mathrm{mU} / \mathrm{min}]$ \\
\hline Basal Period & $0.004(-0.122,0.158)$ \\
2.5-6 hours & $0.091(-0.037,0.224)$ \\
6-12 hours & $0.048(-0.079,0.183)$ \\
Overall & $0.047(-0.093,0.1842)$ \\
\hline
\end{tabular}

Table 4. Residuals of the $S I(t)$ profiles.

\section{DISCUSSION}

The simulated PKs of the composite, hypothetical sensitizer drug were relatively observable in the resulting glucose data using the proposed protocol and identification methods. The variation in $S I(7.9 \%)$ was slightly larger than previous a Monte Carlo study (Lotz et al. 2008). This outcome was an artefact of the reduced sampling rate compared to the test protocol used in that study. Hence, the increased variation was expected and to an extent, validated the other outcomes of this analysis.

The proportional drug effect (D) measurement was particularly stable to noise $(0.9 \%)$. However, the drug absorption $\left(D k_{1}\right)$ and decay rate $\left(D k_{2}\right)$ variables were considerably more susceptible to noise $(116.3 \%$ and $41.4 \%$, respectively). This considerable variation in the drug rate parameters did not have a significant effect on the ability of the protocol and identification method to trace the kinetic and dynamic behaviours of the theoretical drug (Table 4). The variation in the basal insulin sensitivity was comparable to the variation in $S I(t)$. This result implies that parameter trade off occurs. Hence, although the methods presented might not be ideal for the identification of the variables individually,

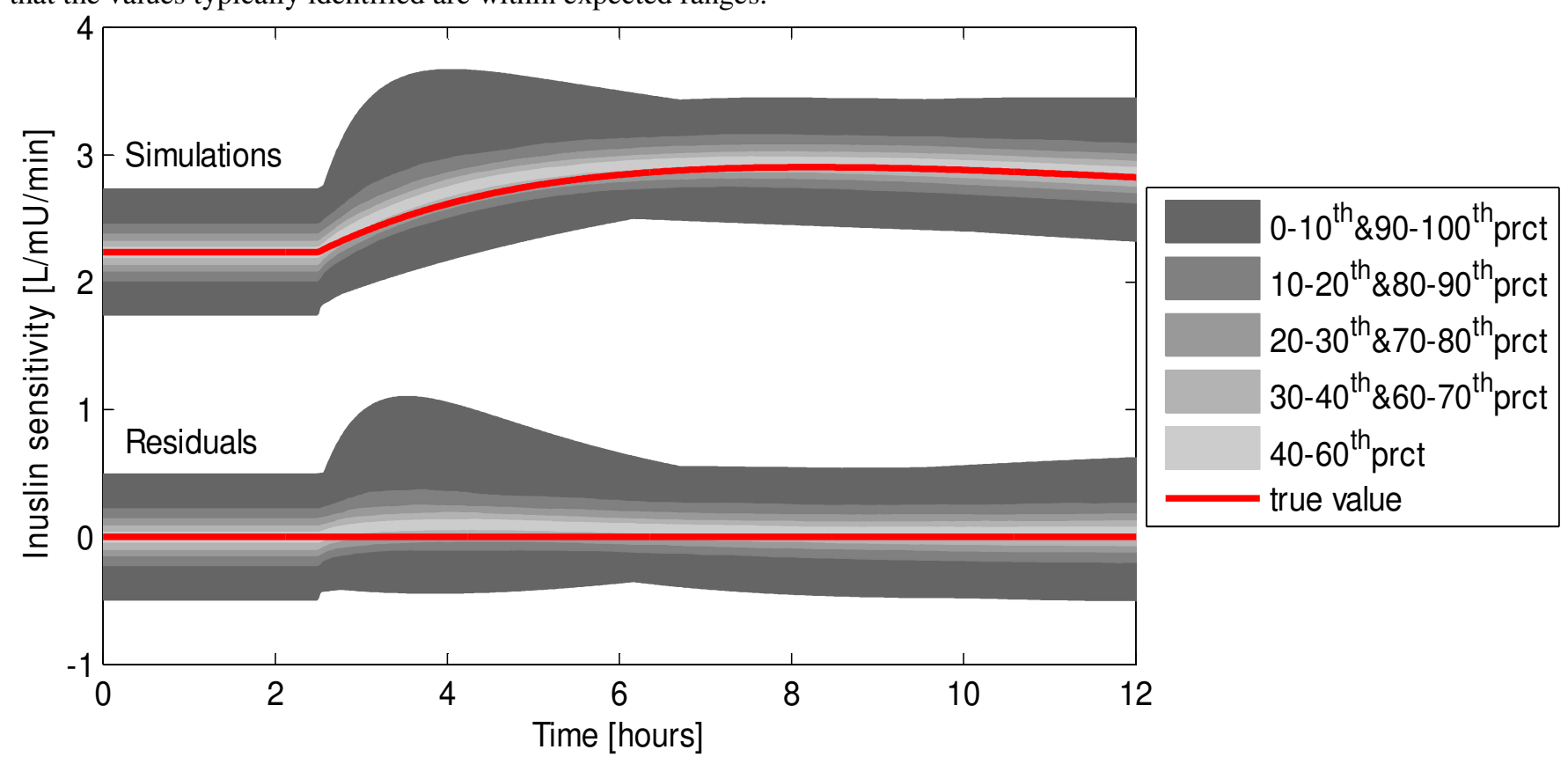

Figure 2. A comparison between the identified insulin sensitivity profiles and the 'true' value of insulin sensitivity for this insilico analysis. 
they may be appropriate for predicting the overall activity of the drug over time. This is the key outcome of such tests as the PKs can be directly measured with direct species assays if desired.

In particular, the findings of this study imply that a clinical pilot investigation of sensitizer kinetics could be undertaken. The existing option for the identification of sensitizer drugs $\mathrm{PD} / \mathrm{PKs}$ is a continuous steady state test such as the euglycemic clamp. This test raises the participant's insulin concentration with a continuous insulin infusion, and euglycaemia is maintained with variable rate of glucose infusion. This variable rate is defined by using feedback control of frequently sampled glucose samples. A specific drug dose will be administered at approximately 2.5-3 hours when the glucose infusion is generally stable. This approach allows a more accurate estimation of the time-varying increase of the insulin sensitivity profile. However, it comes at the cost of significantly increased clinical burden and cost. Table 5 summarizes and compares the attributes of the multiple DISST approach to the existing option for tracing the kinetics and dynamics of insulin sensitizer drugs.

\begin{tabular}{|c|c|c|}
\hline & DISST protocol & EIC protocol \\
\hline Blood samples & 28 & $72-144$ \\
\hline Sample cost (\$NZ) & $\sim 800-1600$ & $\sim 200$ \\
\hline Down-time & $\begin{array}{c}80 \text { minutes } \\
\text { every two hours }\end{array}$ & None \\
\hline Validation & Sparse & Extensive \\
\hline $\begin{array}{r}\text { Physiological } \\
\text { relevance }\end{array}$ & $\begin{array}{l}\text { Within normal } \\
\text { range }\end{array}$ & $\begin{array}{c}\text { Hyper- } \\
\text { physiological }\end{array}$ \\
\hline $\begin{array}{r}\text { Risk of } \\
\text { hypoglycemia }\end{array}$ & Very minimal & Very minimal \\
\hline
\end{tabular}

Table 5. The costs and benefits of the two proposed methods for observing the kinetics and dynamics of insulin sensitizer drugs.

The overall identification method used in this analysis was comprised of two separate types of identification method. Initially, the iterative integral method was used to identify the insulin kinetic variables, and then the $S I$ and $V_{G}$ from the test period prior to drug administration. Following this, nonlinear least squares was used to identify the PK's of the sensitizer drug. The iterative integral method could not identify all five parameters of Equations (9)-(10) as they are not. Furthermore, when the five-variable case was considered, the non-linear lest squares method was either unstable, or unable to converge. As such, the iterative integral method was used to identify $S I$ and $V_{G}$ during the pre-drugdose baseline period, and a change in insulin sensitivity profile is then generated by the drug that allowed a non-linear least-squares identification of the three drug PK and PD variables. This strategy enabled relatively fast, very stable parameter identification. The 1000 simulation Monte Carlo analysis required approximately 3 hours of simulation time and the $0^{\text {th }}$ and $100^{\text {th }}$ percentile shown in Figure 2 show that none of the randomly generated data sets prompted identification failure.

The identification process and the identified values could be further stabilised if one or more of the parameters of Equation (9) could be fixed. For example, the rate of drug absorption $\left(D k_{l}\right)$ may be known, but not the maximal effect $(D)$ or decay rate $\left(D k_{2}\right)$. In this case, the non-linear least square step would only have two variables and variable tradeoff would be limited. Similarly, the decay rate could be predetermined or bounded in separate prior tests.

There were some limitations in this investigation. In particular, the model was contrived for a theoretical drug based on published data and contains simplified PK's that may not fully represent the true PK/PD's of actual sensitizer drugs. Such omitted effects may include:

1. Irreversible transport between the remote and active compartments is not representative of the kinetics of drugs administered subcutaneously. Absorption of the drug if taken orally is irreversible, and the model assumption is valid in this case.

2. The drug may be designed for become stored in fat cells for delayed dispersion. This may be modelled with an added passive third compartment of insulin kinetics.

3. The drug may include combined physiological effects such as:

a. Glucose production suppression. This is not modelled as a time-variant variable in $s$ investigation

b. A combined secretagogue effect. This will be observable with the C-peptide measurement during the trial.

c. Delayed absorption of food. This will not affect dynamic fasting tests, but will be an important attribute of the drug that is not quantified.

4. It is likely that the drug's effect on insulin sensitivity may be saturable, i.e. doubling the bolus may only increase sensitivity by $50-70 \%$ (Laakso et al. 1990, Natali et al. 2000).

5. The drug may require molecular changes that take time to occur. This is not modelled as such, but will be observable 'lumped-in' with the rate of absorption parameter.

All of these factors can be incorporated into the model and so, do not invalidate the findings of this analysis. However, the model appears to be close to the limit of identification in the presence of noise, and thus any further addition would probably require known kinetic behaviours and rates.

Furthermore, the proposed protocol could potentially be used as a replacement for the hyperglycaemic clamp for the identification of the effect of secretagogue drugs. The typical approach of endogenous insulin production identification by a deconvolution of C-peptide data could be used. Parameters for first and second phase insulin production could be obtained from this de-convoluted profile and the variables of 
Equations (6)-(8) could be identified for the case of the secretagogue.

\section{CONCLUSIONS}

The proposed multiple DISST protocol offers a comparatively low-intensity option for the identification of the kinetics and dynamic of insulin sensitizer drugs. The time-varying insulin sensitivity profiles identified in this insilico analysis were quite accurate. However, the variables that defined the profiles showed that identification trade-off was occurring. Thus, although the proposed protocol will enable the identification of the effect of the drug overall, uncertainty exists in the identified drug absorption and decay rate values.

The findings of this study indicate that a pilot trial of this protocol and the identification methods discussed would enable the observation and quantification of insulin sensitizer drugs. However, the next step for this type of test would be an in-silico analysis of a particular drug, instead of the theoretical one discussed here.

\section{REFERENCES}

Docherty P. D., Chase J. G., Lotz T., Hann C. E., Shaw G. M., Berkeley J. E., Mann J. I., McAuley K. A. (2009) DISTq: An iterative analysis of glucose data for low-cost real-time and accurate estimation of insulin sensitivity. Open Med. Inform. J., 3, pages 65-76

Hann C. E., Chase J. G., Lin J., Lotz T., Doran C. V., Shaw G. M. (2005) Integral-based parameter identification for long-term dynamic verification of a glucose-insulin system model. Comput Methods Programs Biomed, 77 (3), pages $259-70$

Laakso M., Edelman S. V., Brechel G., Baron A. D. (1990) Decreased effect of inuslin to stimulate skeletal muscle blood flow in Obese man: A novel mechanism for insulin resistance. J. Clin. Invest., 85 (6), pages 1844-52
Lotz T. (2007) High resolution clinical model-based assessment of insulin sensitivity. PhD Thesis, Department of Mechanical Engineering, University of Canterbury, Christchurch, New Zealand. (247 pages) available at http://hdl.handle.net/10092/1571

Lotz T., Chase J. G., McAuley K. A., Shaw G. M., Wong J., Lin J., Le Compte A. J., Hann C. E., Mann J. I. (2008) Monte Carlo analysis of a new model-based method for insulin sensitivity testing. Computer Methods and Programs in Biomedicine, 89, pages 215-25

Lotz T. F., Chase J. G., McAuley K. A., Shaw G. M., Docherty P. D., Berkeley J. E., Williams S. M., Hann C. E., Mann J. I. (2010) Design and clinical pilot testing of the model based Dynamic insulin sensitivity and secretion test (DISST). J diabetes Sci Technol., 4 (6), pages 1195-201

Natali A., Gastaldelli A., Camastra S., Sironi A. M., Toschi E., Masoni A., Ferrannini E., Mari A. (2000) Doseresponse characteristics of insulin action on glucose metabolism: a non-steady-state approach. Am J Physiol Endocrinol Metab, 278 (5), pages E794-801

Pentikäinen P., Neuvonen P., Penttilä A. (1979) Pharmacokinetics of metformin after intravenous and oral administration to man. European Journal of Clinical Pharmacology, 16 (3), pages 195-202

Van Cauter E., Mestrez F., Sturis J., Polonsky K. S. (1992) Estimation of insulin secretion rates from C-peptide levels. Comparison of individual and standard kinetic parameters for C-peptide clearance. Diabetes, 41 (3), pages $368-77$ 